Archives de sciences sociales des religions

118 | avril - juin 2002

Varia

\title{
Protestantisme évangélique et ségrégation
}

Sébastien Fath

\section{OpenEdition}

Journals

Édition électronique

URL : http://journals.openedition.org/assr/1577

DOI : 10.4000/assr. 1577

ISSN : 1777-5825

Éditeur

Éditions de l'EHESS

Édition imprimée

Date de publication : 1 avril 2002

Pagination : $57-70$

ISBN : 2-222-96718-X

ISSN : 0335-5985

Référence électronique

Sébastien Fath, «Protestantisme évangélique et ségrégation », Archives de sciences sociales des religions [En ligne], 118 | avril - juin 2002, mis en ligne le 14 novembre 2005, consulté le 22 avril 2019. URL : http://journals.openedition.org/assr/1577 ; DOI : 10.4000/assr.1577 


\title{
PROTESTANTISME ÉVANGÉLIQUE ET SÉGRÉGATION
}

\author{
À propos de : \\ EMERSON (Michael O.), SMITH (Christian), Divided \\ by Faith. Evangelical Religion and the Problem of \\ Race in America, Oxford, New York, Oxford \\ University Press, 2000, 212 p. (bibliogr., index, tablx.). \\ GLAUDE JR (Eddie S.), Exodus!Religion, Race, and \\ Nation in Early Nineteenth-Century Black America, \\ Chicago-Londres, Chicago University Press, 2000, 216 p. \\ NEWMAN (Mark), Getting Right with God, Southern \\ Baptists and Desegregation, 1945-1995, Tuscaloosa, \\ University of Alabama Press, 2001, 292 p.
}

Le protestantisme de type " évangélique » (ou Evangelicalism) est souvent présenté, dans ses rapports avec les enjeux sociaux, sous des prismes très contrastés. Il apparaît tantôt sous le signe d'un conservatisme irréductible, quand ce sont les positions de la New Christian Right qui sont mises en avant. Mais il est parfois décrit aussi comme un moteur de non-conformisme culturel aux effets réformistes, émancipateurs. Cette dernière position peut être rapprochée de l'une des grandes thèses d'Ernst Troeltsch, dans Protestantisme et modernité. À ses yeux, c'est le protestantisme de type non-conformiste, spiritualiste et baptiste des courants radicaux de la Réforme qui a en réalité accouché de la modernité ${ }^{1}$ et non pas le protestantisme «classique » issu de la Réforme luthérienne et calviniste, resté ancré dans une conception médiévale des rapports religion-société ${ }^{2}$. Ce qu'il appelle le «nou-

1 "Si, dans son orientation générale, le protestantisme moderne tend vers une liberté de culte indépendante de l'État et si, au sein des Églises, il a voulu ménager un espace au libre jeu de l'esprit et à sa faculté absolument créatrice, de telles finalités n'ont pas été l'objet des grandes confessions, mais en partie du courant baptiste (...) et, pour une autre part, du spiritualisme”. Ernst Troeltsch, Protestantisme et modernité (traduit de l'allemand et préfacé par Marc B. de Launay), Paris, NRF Gallimard, 1991, p. 78.

2 "L'ancien et authentique protestantisme luthérien et calviniste, considéré dans son ensemble, reste, en dépit de sa théorie anti-catholique du salut, entièrement circonscrit par la culture ecclésiastique du Moyen Âge". Ernst Troeltsch, op. cit., p. 47. 
veau protestantisme », caractérisé par une dynamique associative militante et un refus de l'Église d'État, se rapproche à bien des égards de ce que l'on définit aujourd'hui comme le protestantisme évangélique : un protestantisme de conversion où le choix individuel prime sur l'identité par tradition, et où l'engagement associatif local est privilégié sur un encadrement de type "Église-institution ».

Le terrain de l'esclavage et de la ségrégation aux États-Unis ${ }^{3}$ constitue un excellent analyseur de ces enjeux. Il permet de « tester », grandeur nature, l'hypothèse forte de Troeltsch. En effet, l'abolition de l'esclavage puis de la ségrégation peut être comprise dans la perspective d'une modernisation de la société, qui assure la promotion des individus et rompt un communautarisme inégalitaire. Or, on sait que le protestantisme évangélique américain (à forte dominante méthodiste et baptiste) s'est trouvé confronté massivement à cette question esclavagiste. Majoritaire dans le Sud des États-Unis (qui s'est battu pour le maintien de «l'institution particulière » de l'esclavage), très ancré aussi dans les milieux afro-américains où naissent alors les Negro Spirituals, comment a réagi ce type de protestantisme à la « question noire »? A-t-il accéléré la modernisation de la société au travers d'un projet émancipateur, ou a-t-il plutôt défendu une conception "anti-moderne » d'une société stratifiée, ségréguée, inégalitaire ? Trois ouvrages, parmi une très abondante production sur le sujet ${ }^{4}$, permettent d'éclairer en partie cette question.

\section{« Terre Promise » et actualisation politique}

Le livre d'Eddie S. Glaude Jr. développe une analyse fine du recours au thème biblique de «l'Exode " parmi la population afro-américaine de la première moitié $\mathrm{du} \mathrm{XIX}^{\mathrm{e}}$ siècle. Son analyse se focalise essentiellement sur le Nord des États-Unis ${ }^{5}$. Aucune histoire biblique n'a davantage nourri l'imaginaire religieux et politique des Noirs, parce que ce récit d'une libération, du passage de l'esclavage à la «Terre promise », revêtait une redoutable « efficacité politique ${ }^{6}$, donnait aux esclaves, culturellement et géographiquement déracinés ${ }^{7}$, un espoir et une

\footnotetext{
${ }^{3}$ Pour élargir le champ de la réflexion à une comparaison internationale, voir Anthony W. MARX, Making Race and Nation. A Comparison of the United States, South Africa and Brazil, Cambridge, Cambridge University Press, 1998.

${ }^{4}$ Pour une synthèse récente de la production historiographique (en croissance constante depuis vingt ans) sur la religion dans le Sud des États-Unis, voir Donald G. MAthiEws, «We have left undone those things which we ought to have done: Southern Religious History in Retrospect and Prospect», Church History, Studies in Christianity and Culture, 67, 2, juin 1998, pp. 305-325.

5 Pour une analyse symétrique récente dans le Sud, voir Sylvia R. et Betty Wood, Come Shouting to Zion: African Protestantism in the American South and British Caribbean to 1830, Chapel Hill, The University of North Carolina Press, 1998. Dans une perspective plus large, voir aussi : Michael A. GoMEZ, Exchanging our Country Marks: The Transformation of African Identities in the Colonial and Antebellum South, Chapel Hill, The University of North Carolina Press, 1998.

${ }^{6}$ Eddie S. Glaude, JR, Exodus!Religion, Race, and Nation in Early Nineteenth-Century Black America, Chicago-Londres, 2000, p. 3.

${ }^{7}$ Pour le point sur les recherches les plus récentes sur la traite vers les Amériques et leurs conséquences sur les populations déplacées, voir Herbert S. Klein, The Atlantic Slave Trade. New Approches to the Americas, Cambridge, Cambridge University Press, 1999.
} 
« grammaire symbolique » à travers laquelle interpréter leur condition. Le début de l'ouvrage (première partie) est consacré à l'étude des usages du thème de l'Exode dans la construction d'une idée afro-américaine de la nation. L'originalité du propos tient ici dans l'articulation effectuée par l'auteur entre cette genèse identitaire et la construction de la nation américaine dans son ensemble. La thématique biblique de l'Exode, si vivace dans les rangs afro-américains, n'est pas absente non plus, loin s'en faut, chez les white protestants. Dans les populations d'origine européenne, elle a été également mobilisée (quoique d'une manière moins centrale) dans la construction d'une identité nationale ${ }^{8}$. Ce qui dément l'idée d'une disjonction complète, au XIX ${ }^{\mathrm{e}}$ siècle, entre la construction identitaire afro-américaine et celle du reste de la population des États-Unis. Les phénomènes sont liés, raccordés à des références bibliques finalement assez similaires, la différence se jouant moins en terme absolus qu'en terme de degrés d'accentuation.

Ensuite, l'auteur se polarise particulièrement sur le rôle de ce thème dans les débats de la National Negro Convention, depuis sa première réunion (en 1830), jusqu'en 1843, année où la convention décida - à un vote près -, de refuser son soutien à la proposition d'une insurrection des esclaves, formulée par Henry Highland Garnet. Cette structure rassemblait des Noirs libres du Nord, mobilisés pour l'émancipation de leur peuple. En son sein s'observe, selon l'auteur, un véritable continuum entre les expressions religieuses des Black Churches et des revendications a priori séculières : liberté, nouveaux statuts, fin des discriminations. L'auteur l'illustre très bien au travers de l'exemple des trois principales fêtes commémoratives célébrées dans le cadre de la Convention. Le programme suit celui de la liturgie des cultes, incluant chants religieux, lecture de la Bible, sermons, témoignages. Les principaux discours sont dispensés par des prédicateurs, dont les harangues sont infusées de références bibliques, où la rédemption, la "sortie d'Égypte " sont inlassablement invoqués (pp. 85 et ss.). Ces conventions ont constitué, à bien des égards, le premier forum national qui permettait l'engagement civique des populations afro-américaines. Elles auraient développé une « politique de la respectabilité » qui n'est pas seulement à relier au désir d'ascension sociale ${ }^{9}$, mais aussi à l'imaginaire de l'Exode : créant une brèche dans la désespérance, le récit de l'Exode aurait entretenu un esprit de résistance et un sens de la responsabilité, préparant la « sortie de la servitude ». Au contraire d'un « refus utopique des limites » (p. 162), l'imaginaire de l'Exode aurait pris en compte les contraintes concrètes de la servitude tout en préparant méthodiquement, par une prise de conscience graduelle, l'émancipation finale.

On ne peut pas dire que cet ouvrage brille d'une très grande originalité. Après tout, on connaît depuis bien longtemps le rôle considérable joué par les références bibliques (en particulier le thème vétérotestamentaire de la sortie d'Égypte) dans l'imaginaire afro-américain ${ }^{10}$. Mais point n'est besoin d'originalité pour faire un ouvrage de valeur. Eddie S. Glaude Jr. en fournit ici la démonstration, en s’appuyant,

${ }^{8}$ Pour une approche de synthèse, voir l'édition révisée et actualisée de Conrad CHERry, ed., God's New Israel: Religious Interpretations of American Destiny, Chapel Hill, University of California Press, 1998.

${ }^{9}$ Ce que souligne Evelyn Brooks Higginbotham dans Righteous Discontent, The Women's Movement in the Black Baptist Church, 1880-1920, Cambridge-Londres, Harvard University Press, 1993.

${ }^{10}$ Cf. par exemple Mickaël Waltzer, Exodus and Revolution, New York, Basic, 1985. 
en bon historien, sur un corpus de sources bien délimité, et exploité rigoureusement. Ce que l'auteur s'attache particulièrement à montrer, c'est la relation entre imaginaire religieux, expression cultuelle et actualisation politique. En effet, c'est par le culte, le chant, la prière, la vie communautaire (dans des Églises, surtout méthodistes et baptistes, que l'on peut rattacher au protestantisme évangélique) que les esclaves ont pu actualiser, dans leur existence concrète, les thèmes bibliques rattachés à l'Exode. C'est par leur engagement religieux, par leur vie cultuelle intense qu'ils ont pu rompre la distance utopique entre le temps biblique de l'esclavage en Égypte et leur condition présente d'esclaves africains aux États-Unis, effectuant une sorte de fusion identitaire avec les «enfants d'Israël» opprimés. Cette fonction d'actualisation politique se doubla aussi d'un processus de construction d'une identité " nationale ». Le récit biblique de l'Exode n'est en effet pas seulement le récit de la libération d'un certain nombre d'individus opprimés. Il est aussi, et peut-être surtout, le récit de la naissance d'un peuple, de la naissance d'une nation, forgée dans l'expérience inaugurale de l'émancipation, de la " sortie d'Égypte ». L'auteur montre bien comment le récit de l'Exode a constitué, en plein $\mathrm{XIX}^{\mathrm{e}}$ siècle, la principale "métaphore » au travers de laquelle les afro-américains se sont construit une conscience collective. C'est au travers du vecteur religieux du christianisme que cet engagement politique s'est affirmé.

Il se distingue en partie, sur ce terrain, du livre d'Eugene Genovese ${ }^{11}$. Pour ce dernier, l'identité chrétienne évangélique des afro-américains a globalement freiné le processus de revendication politique collective. En effet, esclaves et maîtres fréquentaient des Églises similaires (souvent baptistes et méthodistes). Les oppresseurs étaient donc aussi, à certains égards, des "frères et sœurs », en dépit du prodigieux contentieux qui séparait les deux populations. Par ailleurs, les préceptes moraux du christianisme évangélique interdisaient une perspective révolutionnaire violente, et auraient donc globalement ralenti l'émergence d'une conscience « nationale» noire. Pour Eddie S. Glaude Jr, Genovese fait en partie erreur sur ce plan. L'imaginaire de l'Exode aurait en effet directement nourri la construction identitaire et politique du «peuple » afro-américain » en marche vers la liberté. Il en aurait même constitué l'aliment principal, montrant par là comment l'inculturation du christianisme de type évangélique en terrain afro-américain a pu conduire à des effets d'émancipation considérables. Si la morale chrétienne a cependant joué parfois comme frein, ce n'est pas sur le terrain de l'émancipation ni du politique (bien au contraire), mais sur celui de la violence physique, l'ethos chrétien partagé avec les maîtres encourageant plutôt un combat d'ordre symbolique, au sein d'une culture chrétienne largement commune, afin de réorienter les interprétations dominantes dans un sens libérateur. En prolongeant la réflexion de l'auteur, on peut considérer que la culture chrétienne des populations afro-américaines de la première moitié du XIX $\mathrm{X}^{\mathrm{e}}$ siècle, à forte connotation " évangélique », paraît bien avoir joué dans le sens d'une dynamique émancipatrice, face au conservatisme d'une société encore favorable à la ségrégation et à l'esclavage. Mais comment comprendre alors les résistances parfois très vives opposées par les Blancs, y compris quand ils se rattachent au protestantisme évangélique ? Comment l'imaginaire de rédemption, de renaissance ou "nouvelle naissance », si typiquement évangélique, a-t-il pu, dans un cas, alimenter une dynamique d'émancipation politique, et dans

\footnotetext{
${ }^{11}$ Eugene Genovese, Roll, Jordan, Roll: The World the Slaves Made, New York, Vintage, 1976.
} 
l'autre, nourrir un conservatisme ségrégationniste ? C'est à cette question que s'attèle, pour la période 1945-1995, Mark Newman.

\section{Ségrégation : débats baptistes et poids du populisme}

À partir d'un dossier très différent de celui d'Eddie Glaude Jr. (non plus la première moitié du $\mathrm{XIX}^{\mathrm{e}}$ siècle, mais la seconde moitié du $\mathrm{XX}^{\mathrm{e}}$ siècle, non plus le christianisme afro-américain, mais celui des Blancs du Sud des États-Unis), Marc Newman s'interroge sur les relations entretenues par les baptistes du Sud (rattachés à la Southern Baptist Convention -SBC-, la plus grande dénomination protestante des États-Unis) avec la ségrégation. Cette période conduit d'un paysage religieux ${ }^{12}$ et social fortement compartimenté, vers une "intégration raciale » progressive, incomplète et douloureuse. Newman focalise son analyse sur les onze anciens États confédérés, à l'aide (en particulier) d'un important dépouillement de la presse dénominationnelle. Des réactions des conventions de chaque État à celles des collèges, des Églises locales, en passant bien-sûr par le sommet de l'appareil dénominationnel, tous les terrains sont investigués. Depuis la Civil War jusqu'à l'arrêt Brown vs. Board of Education de la Cour Suprême, la SBC a massivement soutenu la ségrégation ambiante, tout en défendant une ligne fortement évangélique, conversionniste (teintée d'orientations parfois fondamentalistes). Jusqu'à présent, la SBC n'était pas réputée, pour la rapidité de ses évolutions, après 1954. La ségrégation, voire même l'esclavage (mais sotto voce), apparaissaient comme justifiables bibliquement. N'a-t-il pas fallu attendre 1995 pour que la SBC demande publiquement pardon pour son soutien passé à l'esclavage et à un certain racisme ambiant ? Il est vrai que les autres dénominations chrétiennes du Sud n'ont globalement pas fait preuve d'un grand zèle dans le soutien à la politique de déségrégation ${ }^{13}$ (loin s'en faut), mais dans ce conservatisme général, la Southern Baptist Convention faisait figure de modèle de résistance au changement. Pourtant, Marc Newman s'attache à montrer que les réactions de la SBC n'ont pas été monolithiques, et qu'un vif débat a traversé ses rangs sur la question de la place des Afro-américains dans la société. «Jusqu'au milieu des années 1960, la majorité des sudistes blancs et des baptistes du Sud étaient en faveur de la ségrégation ${ }^{14}$, mais non sans tensions et controverses. Trois principaux groupes sont distingués: une minorité conservatrice qui défend un " jusqu'au boutisme » de la ségrégation au nom de la Bible, une majorité modérée qui considère la ségrégation comme acceptable, dans la mesure où elle

${ }^{12}$ Sur la constitution des Black Churches, après la Guerre Civile américaine, voir notamment William E. Montgomery, Under Their Own Vine and Fig Tree: The African-American Church in the South 1865-1900, Baton Rouge, Louisiana State University Press, 1993.

${ }^{13}$ Pour une étude de cas sur le terrain méthodiste, voir Donald E. Collins, When the Church Bell Rang racist: The Methodist Church and the Civil Rights Movement in Alabama, Macon, Mercer University Press, 1998.

${ }^{14}$ Mark Newman, Getting Right with God, Southern Baptists and Desegregation, 1945-1995, Tuscaloosa, University of Alabama Press, 2001, p. 20. 
«fait partie du paysage » social, et une minorité progressiste de responsables de l'appareil SBC, de laïcs, de pasteurs, qui conteste, puis rejette ouvertement la ségrégation, comme totalement contraire à l'Évangile. Cette minorité, où les étudiants ont pesé d'un poids significatif (pp. 164 et ss.) a graduellement entamé la " structure de plausibilité " des conceptions ségrégationnistes, par le biais de son activité éditoriale, des commissions Social Service et Christian Life, et des séminaires.

La démonstration de Marc Newman séduit par sa rigueur, l'abondance de la documentation traitée, mais elle paraît pousser un peu trop loin l'accent sur la sensibilité progressiste. Autant la réflexion paraît totalement convaincante lorsqu'elle expose la force des débats internes, autant elle apparaît plus fragile lorsqu'elle souligne l'impact et les progrès des anti-ségrégationnistes. En fondant une large part de sa documentation sur la presse dénominationnelle, Newman paraît avoir tendance à surévaluer un peu (même s'il est conscient du risque) le point de vue des responsables, au détriment des «laïcs » de base. Or, il semble bien que la position de ces membres ordinaires ait été globalement très attentiste, conservatrice, comme l'auteur le reconnaît d'ailleurs au passage (mais sans insister suffisamment) en soulignant que leur attitude diffère peu de celle des autres sudistes blancs. A l'occasion d'un dépouillement de Christianity Today, le principal périodique évangélique américain, la lecture d'un courrier d'un «pasteur de base » (de la Warren Baptist Church de Martinez, Georgie) paraît souligner ce décalage entre la « base » conservatrice et des élites plus ouvertes. Le pasteur baptiste en question réagit (en 1957) à un texte du Dr Henlee Barnett, un théologien des Southern Baptists (curieusement oublié par Newman), qui estime que tous les prédicateurs baptistes du Sud pensent que la ségrégation n'est pas chrétienne. Le pasteur réagit très vigoureusement : «il argumente sur la base d'une fausse généralisation. Beaucoup d'entre-nous pensent que la ségrégation n'est ni non-chrétienne, ni non-démocratique ». Et l'auteur de continuer en estimant que les textes bibliques cités en faveur de l'intégration «manquent à la fois de clarté et d'étoffe ${ }^{15}$. Tout en reconnaissant ce type de résistance, Mark Newman paraît légèrement manquer l'équilibre entre un légitime souci d'éviter la caricature («tous les baptistes du Sud ont été ségrégationnistes») et le risque d'une image légèrement édulcorée des positions tenues. Ce qui ressort massivement, in fine, est la position «attestataire » et non "contestataire » des baptistes du Sud. Ils sont certes traversés par des débats internes assez vifs, très bien restitués (et situés) par Newman, mais ils restent du côté du «parti de l'ordre » et de la «tradition ». Ils ne suivent le chemin de la déségrégation qu'avec une certaine lenteur. C'est véritablement dans les vingt dernières années du $\mathrm{XX}^{\mathrm{e}}$ siècle, alors qu'ils connaissent par ailleurs une crise d'identité aux enjeux complexes ${ }^{16}$, qu'ils paraissent accepter massivement "l'intégration raciale», au sein d'une société du Sud (et d'un protestantisme) en mutation ${ }^{17}$. Il reste qu'à la fin du $\mathrm{XX}^{\mathrm{e}}$ siècle, si la SBC ne compte plus, dans ses rangs, d'apologètes de la

15 A. H. Simmons, Christianity Today, 25 novembre 1957, vol 2, n 4, p. 27.

${ }^{16}$ Cette crise, marquée par une affirmation néo-fondamentaliste au sein (et à la tête) de la dénomination, a été remarquablement éclairée par Nancy Tatom AMMERMAN, in Baptist Battles: Social Change and Religious Conflict in the Southern Baptist Convention, New Brunswick, N.J., Rutgers University Press, 1990.

17 Voir Marion D. Albridge et Kevin Lanis, eds., The Changing Shape of Protestantism in the South, Macon, Mercer University Press, 1996. 
ségrégation, Marc Newman rappelle qu'elle demeure fortement clivée. Les Églises baptistes «mixtes » (Blancs et Noirs assis sur les mêmes bancs) restent l'exception.

Newman livre en conclusion une clef majeure de cet attentisme des baptistes du Sud sur la question noire : "si la SBC avait été moins démocratique et plus hiérarchique, et par là même, moins vulnérable » à l'intervention de la base ségrégationniste, «elle aurait évolué plus vite et plus loin sur le terrain des relations raciales sous l'influence d'une élite progressiste » (p. 209). En d'autres termes, sa structure congrégationaliste, valorisant l'autonomie de l'assemblée locale, le choix individuel et la démocratie communautaire (connotés, en principe, comme «facteurs de modernité »), a favorisé l'emprise de réflexes populistes, puissamment conservateurs, obstacles au changement ${ }^{18}$. Quand la sociabilité communautaire de type évangélique permettait aux esclaves noirs du début du XIX ${ }^{\mathrm{e}}$ siècle d'actualiser une utopie libératrice, force de contestation de l'ordre établi, on voit donc, symétriquement, qu'un même type de sociabilité (dans des Églises qui sont souvent baptistes, aussi bien en terrain afro-américain qu'en terrain " petit blanc ») a nourri un populisme conservateur, freinant les dynamiques plus contestataires d'une «élite» dénominationnelle formée dans les séminaires. Dans un cas comme dans l'autre, cependant, un point commun reste le débat: dans la National Negro Convention comme dans la Southern Baptist Convention, il semble que la culture religieuse ambiante ait provoqué des discussions particulièrement vives, clivées, où la variété des points de vue apparaît comme «naturelle » en dépit d'une lutte serrée pour l'hégémonie idéologique.

\section{Évangéliques aujourd'hui : l'impossible mixité ethnique?}

Au-delà du terrain particulier des baptistes du Sud, on peut se demander, plus largement, quelle a été l'attitude des protestants évangéliques dans leur ensemble (du Sud comme du Nord) face au "problème racial » hérité des temps de l'esclavage et de la ségrégation. Mais la question n'est-elle pas trop vaste ? Un ouvrage s'est pourtant attaché à la prendre à bras le corps. Salué par l'historien Mark Noll comme « le meilleur ouvrage publié sur les attitudes raciales des white evangelical Protestants $\gg{ }^{19}$, il a suscité une très vive attention du monde scientifique, mais aussi de la société américaine dans son ensemble. Sur la page de couverture figurent les photographies en parallèle de deux chapelles évangéliques, l'une occupée exclusivement par des Noirs, l'autre par des Blancs. Avec le constat suivant: « divisé par la foi ». Le sous-titre, qui complète la thématique, est en partie trompeur : il suggère que le sujet traité sera «la religion évangélique et le problème racial en Amérique ». En réalité, le but du livre est plus modeste. Il ne traite pas de

18 À l'inverse, des Églises plus hiérarchisées, comme l'Église presbytérienne ou l'Église catholique, ont évolué un peu plus vite que la SBC sur la question raciale même si, dans le Sud, des freins culturels ont joué partout. L'auteur le montre dans le chapitre 10 : «The Major White Denominations and Race Relations, 1945-1971», M. NewmAn, op. cit., pp. 168-190.

${ }^{19}$ Mark Noll, cité en page de dos de l'ouvrage. 
la question « raciale » en général, mais des relations entre Noirs et Blancs (terrain déjà considérable !). Une lecture attentive de l'ouvrage conduit à resserrer encore le champ : c'est essentiellement de la perception des Noirs par les protestants évangéliques blancs dont il est question. «Le gouffre qui sépare les Noirs américains des Blancs est globalement plus vaste, et l'histoire plus longue », que dans le cas des autres clivages ethniques ${ }^{20}$. Les auteurs, Michael O. Emerson (Rice University) et Christian Smith (Chapel Hill University), font le constat suivant : en dépit des bonnes intentions manifestées depuis une vingtaine d'années au sein du mouvement évangélique, par la voix de leaders comme Billy Graham ou d'organisations comme les Promise Keepers ${ }^{21}$, la « réconciliation raciale » n'a pas abouti, loin s'en faut. Suivant une formule choc de l'ouvrage, le dimanche matin à 11 heures est une des heures où la ségrégation est la plus forte aux États-Unis. Blancs et Noirs se dirigent dans leurs Églises, sans se mélanger. Les auteurs s'appuient, dans leur analyse, sur une enquête nationale par téléphone conduite auprès de 2000 individus issus du protestantisme évangélique «blanc», et sur deux-cents entretiens approfondis.

Le début de l'ouvrage est constitué par des considérations de définition et de méthodologie. Le protestantisme évangélique (evangelicalism) est présenté comme « la religion dominante (mainstream) durant les 125 premières années de l'histoire américaine » (p. 3). Le protestantisme évangélique est ici défini avant tout suivant des critères de doctrine (crucicentrisme) et d'engagement (rôle majeur du témoignage), ce qui conduit par exemple les auteurs à présenter des hommes comme Cotton Mather ou Jonathan Edwards, dans le chapitre 2, comme "évangéliques », choix qui ne fait pas tout à fait l'unanimité parmi les historiens. Une difficulté de leur perspective (déjà présente dans deux ouvrages précédents de Christian Smith) est que la définition du terme " évangélique » repose largement sur l'auto-identification. Une définition sociologique doit certes prendre en compte les formulations données par les acteurs eux-mêmes. Mais est-ce suffisant ? Que les «évangéliques » se définissent eux-mêmes sur des bases d' " orthodoxie engagée » ne devrait pas obligatoirement conduire le chercheur à reprendre ces critères sans les combiner avec des variables plus objectivées (par exemple, le type de sociabilité religieuse mise en œuvre). Par ailleurs, des acteurs qui se définissent eux-mêmes comme "évangéliques » ne le sont pas obligatoirement au regard d'une définition construite par le sociologue. Inversement, des acteurs qui ne se qualifient pas d'évangéliques peuvent parfaitement correspondre aux critères retenus par le chercheur : par exemple, les baptistes ou méthodistes afro-américains n'auront pas tendance à se définir comme « évangéliques », terminologie qui, à leurs yeux, renvoie à l'univers des « Blancs ». Smith et Emerson, du coup, peuvent affirmer (p. 3) que " presque $90 \%$ des Américains qui se définissent eux-mêmes comme évangéliques sont blancs ». Mais un tel constat ne revient pas à dire que neuf évangéliques américains sur dix sont blancs. Or, Smith et Emerson ont une tendance certaine à «coller» à l'auto-définition des « évangéliques », ce qui peut poser des difficultés dans la mise en perspective.

${ }^{20}$ Michael O. Emerson et Christian Smith, Divided by Faith. Evangelical Religion and the Problem of Race in America, Oxford, New York, Oxford University Press, 2000, p. 11.

${ }^{21}$ Plus de trente pour cent du staff des Promise Keepers serait composé d'Afro-Américains (EMERSON et SMith, op. cit., p. 65). 
Cette réserve de méthode mise à part, les données d'enquête sont traitées avec une rigueur exemplaire (après un chapitre historique d'excellente facture, pp. 21 à 49). Elles éclairent comme jamais auparavant les nœuds culturels qui résistent à la " mixité raciale ». L'individualisme évangélique ressort massivement des entretiens. La libre entreprise, les relations inter-personnelles, le choix individuel, se combinent, chez eux, à un «anti-structuralisme» viscéral (p. 76) : les protestants évangéliques refusent généralement la prise en compte des explications sociales structurelles au profit d'une grille essentiellement psychologique. Tout est passé au crible de la volonté et de la morale individuelles. Même si certains interviewés admettent qu'il existe une certaine discrimination institutionnelle, celle-ci est attribuée à des erreurs ou des fautes individuelles, et non pas à un problème structurel, enkysté dans l'institution elle-même ${ }^{22}$. L'idée de " fait social » selon Durkheim apparaît aux antipodes des représentations des évangéliques, pour lesquels la société ne s'explique que par l'individu, selon ce slogan publicitaire publié dans le mensuel Christianity Today, et que citent Smith et Emerson : "si vous voulez un monde meilleur, composé de meilleures nations, habité par de meilleurs États, remplis de meilleurs comtés, constitués de meilleures cités, qui comprennent de meilleurs voisinages, illuminés par de meilleures Églises, peuplées par de meilleures familles, alors vous avez à commencer par devenir une meilleure personne » ( $\mathrm{p}$. 115). À cet individualisme principiel, qui rend aveugle aux explications sociales plus larges, s'ajoutent les habitudes de choix, excellemment mises en perspective dans le chapitre 7 de l'ouvrage. Les auteurs pointent à quel point l'offre et la demande religieuses structurent aux États-Unis un "marché religieux » concurrentiel où les individus effectuent en permanence des choix d'appartenance en fonction de leurs préférences personnelles. S'inscrivant dans la perspective de Nathan Hatch $^{23}$, Roger Fink et Rodney Stark ${ }^{24}$, les auteurs soulignent combien le protestantisme évangélique a contribué au basculement d'une religion « établie » (à choix individuel restreint) vers un marché ouvert à des entrepreneurs religieux créatifs, transformant les États-Unis en «mega mall 25 » religieux (p. 139). Cette logique consumériste constitue, aux yeux de Smith et Emerson, un facteur majeur d'explication de l'homogénéité sociale (et « raciale ») de la grande majorité des Églises évangéliques américaines. La logique du choix individuel a en effet conduit à la constitution de "niches », c'est-à-dire des segments de population sensibles aux mêmes besoins. Les congrégations se superposent de plus en plus étroitement à ces « niches », peuplées d'individus de statut socio-économique similaire, d'origine ethnique comparable, voire même d'âge assez proche. Car le "coût» demandé à l'individu est dans ce cas moins grand (en terme d'adaptabilité, en particulier) que dans le cas d'une assemblée à grande diversité interne (pp. 144-145).

\footnotetext{
22 Dans une des multiples tables statistiques proposée en annexe, les auteurs présentent un relevé d'occurrences effectué dans le mensuel évangélique Christianity Today entre 1994 et 1998. parmi les solutions alternatives au racisme, vingt occurrences mentionnent "connaître des gens de l'autre race". Seules deux occurrences évoquent "travailler contre la discrimination à l'emploi et dans la justice" (p. 179)

${ }^{23}$ Nathan Hatch, The Democratization of American Christianity, New Haven, Yale University Press, 1989.

${ }^{24}$ R. FInKE et R. STARK, The Churching of America 1776-1990. Winners and Losers in our Religious Economy, New Brunswick, Rutgers University Press, 1992.

25 Aux États-Unis, un "mall" est un immense centre commercial pouvant rassembler plusieurs centaines de commerces, souvent en périphérie des centres urbains.
} 
Un exemple saisissant ressort des entretiens conduits par les deux sociologues. Il s'agit d'une congrégation que les auteurs appellent, par convention, la «First Baptist Church» (pp. 147-150). Un jeune pasteur évangélique afro-américain nouvellement installé à Seattle, se sent appelé à développer une Église locale inter-raciale. Il dispose pour cela de l'appui de sa dénomination et d'un haut degré de motivation. Conscient du fait que les congrégations recrutent des membres en fonction de leurs réseaux de relation (networks), et que ces réseaux tendent à une certaine homogénéité sociale et ethnique, le pasteur décida donc de commencer d'entrée avec une assemblée mixte, composée de gens déterminés à la réussite d'une telle entreprise. Il visita, dans ce but, plusieurs Églises évangéliques « blanches » et " noires », demandant aux familles motivées d'accepter de quitter leur assemblée pour développer son projet d’Église «mixte ». Après une année de préparation, l'assemblée put démarrer, sur la base d'une mixité presque parfaite : cinquante pour cent de Blancs, cinquante pour cent de Noirs. Cette proportion se retrouvait dans l'encadrement, l'orchestre, la chorale... Mais, tandis que la nouvelle Église commença à se développer, la mixité recula. Après un an, une famille blanche décida de partir. Peu de temps après, une autre famille blanche fit de même. De moins en moins de Blancs extérieurs à la congrégation visitèrent l'Église. Peu à peu, les Blancs qui restaient éprouvèrent un malaise. Ils considéraient qu'en dépit des efforts authentiques conduits par le pasteur et l'équipe d'encadrement, leurs attentes n'étaient pas suffisamment prises en compte. D'autres partirent. Au bout de trois ans d'expérience "mixte», il ne restait plus que dix membres blancs. Les entretiens conduits avec les sociologues révélèrent que tous les dix envisageaient de partir. Pourquoi ce phénomène ? L'explication ne peut correspondre à un manque de motivation, au contraire. Tous les membres ont délibérément choisi de tenter le pari de la mixité. Elle ne peut pas non plus renvoyer à un manque de "bonne volonté ». La logique "let's be friends » (titre du chapitre 6 de l'ouvrage) animait en effet tous les participants. C'est au-delà des questions individuelles et inter-personnelles (systématiquement mises en avant par les évangéliques) que l'on peut trouver l'explication. Les entretiens oblitèrent complètement les éléments d'explication macro-sociales. Tous les interviewés clament que leur choix de partir n'a rien à voir avec la « race», mais tout avec leurs «besoins » non satisfaits. Ce qui les a tous conduits à rejoindre des Églises très majoritairement «blanches ».

Smith et Emerson avancent deux éléments d'explication, reliés aux logiques du « marché religieux » américain. Le premier facteur explicatif est qualifié de "niche edge effect» (littéralement, l'effet «bord de la niche »). Dans les associations volontaires, les personnes situées un peu en périphérie de la «niche » subiront un turnover plus important que ceux qui sont au centre, parce que les liens extraassociation sont plus importants que les liens intra-association. Le départ des premières familles blanches a très rapidement créé un sentiment de marginalisation chez ceux qui restèrent, sentiment d'autant plus fortement ressenti que les Blancs (au contraire des Noirs) ont culturellement l'habitude de la majorité. La mixité " parfaite » constituait déjà, en soi, une rupture par rapport à cette position dominante. Le départ de la première famille accentua le sentiment d'inconfort, de marginalité, entraînant un effet de spirale et une succession de départs. Le second facteur explicatif invoqué (que les auteurs empruntent, comme le premier, à Pamela Popielarz 
et J. Miller Mc Pherson ${ }^{26}$ ) est celui de « niche overlap effect » (littéralement : « effet de chevauchement entre niches »). Chaque Église qui tend à fonctionner en tant que « niche » empiète sur le territoire de l'autre, à la recherche de membres potentiels. Les membres blancs de l'Église mixte sont dès lors confrontés au moins à deux types d'offre : celle de l'Église mixte dans laquelle ils ont initialement décidé de s'engager (où ils éprouvent un sentiment diffus de "périphérie » - le niche edge effect -) mais aussi celle, plus « classique » et plus « confortable », d'Églises évangéliques blanches homogènes. Ce chevauchement des offres, dont une est plus atypique que les autres, va graduellement user la détermination des Blancs à demeurer dans l'Église mixte. L'offre concurrente, plus « classique », où ils ne subiront pas le « niche edge effect» puisqu'ils seront « entre Blancs », tend progressivement à l'emporter. La combinaison de ces deux effets tend presque systématiquement à constituer des assemblées évangéliques ethniquement homogènes - alors même qu'explicitement, les acteurs ne paraissent pas le souhaiter -. Emerson et Smith concluent justement en soulignant que la disparition de sentiments racistes, l'extinction d'une pensée de la ségrégation, la bonne volonté favorable à la mixité, ne suffisent pas à générer des assemblées mixtes. Les effets induits par les logiques du marché religieux, et ses " niches » de fidèles, entraînent " mécaniquement » des assemblées globalement homogènes. Les acteurs ne parviennent pas à inverser la tendance, car leur refus quasi général d'aborder des causes macro-sociales les confine dans un registre explicatif inopérant (le psychologisme, les relations inter-individuelles). Faire face à la question raciale suppose de « déplacer des barrières structurelles» (p. 172), ce que les white evangelicals n'envisagent pas. $\mathrm{Ne}$ parvenant pas à saisir les causes sociales de la «division raciale » persistante, les protestants évangéliques blancs entretiennent donc le statu quo, en dépit de la meilleure « bonne volonté ». La conclusion des auteurs balance entre pessimisme et espoir. Quittant le terrain strictement scientifique pour interpeller le milieu protestant évangélique «blanc », Emerson et Smith invitent à une prise en compte beaucoup plus vive des sciences sociales, des explications structurelles. En d'autres termes, les protestants évangéliques sont en quelque sorte invités à modifier leur « activisme à courte vue ${ }^{27}$ (p. 171)... et à lire Durkheim !

\section{Un rapport ambivalent à la modernité}

À leur manière, les ouvrages de Eddie S. Glaude Jr, Emerson et Smith aboutissent à la fois à un constat similaire, et à des conclusions apparemment opposées. Ces livres, qui abordent tous trois le rapport entre un christianisme protestant de type "évangélique » et la question raciale, soulignent tous la vivacité du débat interne. La National Negro Convention constitua un forum d'un grand dynamisme,

\footnotetext{
${ }^{26}$ Pamela Popielarz et J. Miller Mc Pherson, «On the Edge or in Between: Niche Position, Niche Overlap, and the Duration of Voluntary Association Memberships», American Journal of Sociology, 101, pp. 698-721.

${ }^{27}$ Les auteurs s'appuient sur un ouvrage de l'historien Mark NoLL, qui fit grand bruit en 1994 : M. Noll, The Scandal of the Evangelical Mind, Grand Rapids, Eerdmans, 1994. L'auteur y analyse les raisons de fond de la faiblesse intellectuelle du milieu évangélique américain.
} 
où la mythologie émancipatrice de l'Exode, portée par les Black Churches, trouva un écho et un terrain d'actualisation privilégié. La Southern Baptist Convention, nous apprend Mark Newman, fut beaucoup moins monolithique qu'on aurait pu l'imaginer sur la question de la ségrégation après 1945 : les débats y firent rage, particulièrement entre une "base » conservatrice et des "élites » beaucoup plus favorables au changement. Quant au terrain étudié par Emerson et Smith, il révèle aussi du débat, de l'échange, du volontarisme. Quoique confinée et réduite à un registre psychologique et moral, la question raciale n'apparaît pas tabou. Les interviewés l'évoquent volontiers ; elle est discutée dans les Églises et les mouvements para-church: Bill Mac Cartney, cité par Emerson et Smith, rapporte qu'après la conférence de 1996 du mouvement qu'il préside (les Promise Keepers), "presque $40 \%$ de ceux qui se sont plaints ont évoqué la mention de la réconciliation raciale » (p. 68), thème qui connaît une «explosion », dans les milieux évangéliques (p. 59). Signes évidents d'un débat dont la montée en puissance est retracée dans le chapitre 3 ( "Becoming Active »). Les assemblées évangéliques paraissent donc globalement comme des lieux d'interaction dynamiques, où des relations sociales intenses s'opèrent et des conflits de valeur sont mis en scène. En revanche, les ouvrages recensés paraissent diverger radicalement dans les implications de ces logiques sociales. Dans le cas du milieu évangélique noir, l'effet paraît de toute évidence avoir joué dans un sens d'émancipation, brisant les logiques de soumission et de déshumanisation au profit d'un projet d'accès à une individualité socialement reconnue et aux droits sociaux, «terre promise » du combat. Dans le cas de la Southern Baptist Convention, en revanche, la sociabilité évangélique a essentiellement offert une «caisse de résonance » au populisme ambiant, encourageant un attentisme social clairement réticent face au progressisme volontariste du Civil Rights Movement. Enfin, le dossier traité dans Divided By Faith tend à montrer que la culture individualiste des protestants évangéliques contemporains les aveugle aux rapports de domination, aux enjeux sociaux globaux, perpétuant une situation de non-mixité en dépit des «bons sentiments » affichés.

Ces trois lucarnes ouvertes sur les relations entre protestantisme évangélique et question raciale aux États-Unis permettent un réexamen, in fine, de la vieille hypothèse de Troeltsch. Il semble que l'on puisse formuler la conclusion suivante : le néo-protestantisme » non conformiste, de type baptiste et spiritualiste, que Troeltsch décrit comme agent de modernisation, du XVI ${ }^{\mathrm{e}}$ au XVIII ${ }^{\mathrm{e}}$ siècle, n'est pas intrinsèquement modernisateur. Il n'apparaît pas possible de généraliser l'interprétation troeltschienne, ou de la «tirer » dans un sens systématique. Que le protestantisme évangélique, par son choix de séparation des Églises et de l'État, son accent sur l'individu et la démocratie locale, ait pu, dans un temps donné (en l'occurrence, à l'époque moderne, "pré-1789 ») générer des effets de modernité, soit. Mais il apparaît erroné d'établir un lien intrinsèque entre le type social « évangélique » et la modernité (ou les « effets de modernité »). Si l'époque contemporaine (à partir du XIX ${ }^{\mathrm{e}}$ siècle) peut parfois faire écho aux hypothèses troeltchiennes (cas du christianisme évangélique afro-américain par exemple), elle les dément également dans bien des cas. Le dossier des baptistes du Sud apparaît exemplaire : c'est bel et bien dans le sens d'un statu quo conservateur, perpétuant le plus longtemps possible une ségrégation archaïque, qu'a pesé la «base » baptiste. L'effet de 
majorité a sans doute joué ici ${ }^{28}$ (les Églises baptistes devenant, sans le reconnaître, proches d'une «Église établie », plus « attestataire » que « contestataire »), mais aussi le caractère démocratique et local des assemblées. Révolutionnaire et progressiste dans un contexte comme celui de l'Europe du XVII ${ }^{e}$ siècle (où la logique holiste était de règle), ce caractère peut nourrir, dans une société démocratique, de redoutables effets populistes et conservateurs ${ }^{29}$, le pasteur ou le théologien « éclairé » pouvant se retrouver complètement prisonnier d'une " base » conservatrice, sans le recours qu'il est possible d'obtenir «par le haut» dans une Église comme l'Église catholique, par exemple. L'individualisme lui-même, aux effets si « modernes », il y a deux siècles de distance, peut jouer dans un sens sinon « anti-moderne », du moins « conservateur » dans un marché religieux de type américain, comme le montrent très bien Emerson et Smith. On le sait : il y a individualisme et individualisme! Si l'individualisme «évangélique » se détermine uniquement en fonction de choix de « confort », nourrissant des « niches » affinitaires (sur le modèle des logiques de marketing), l'homogénéité sociale des associations de convertis peut fort bien conduire à des formes de lien social moins proches de l'idéal moderne du melting-pot que d'un «néo-communautarisme » où l'identique appelle l'identique.

\section{Sismographes du changement social ?}

En conclusion, l'hypothèse d'un protestantisme évangélique aux effets intrinsèquement modernisateurs ne semble pas pouvoir être retenue. À la lumière des trois ouvrages recensés ressort une réalité contrastée. À la modernisation observée sur certains terrains répond le conservatisme traditionnel constaté ailleurs - massivement. En revanche, le dossier de l'esclavage et de la discrimination raciale révèle la forte capacité de débat et de réaction (sans préjuger de son sens) des Églises évangéliques. C'est dans leurs rangs, plus prestement et plus vigoureusement que dans les grandes Églises ${ }^{30}$, qu'un débat s'est épanoui, que des clivages se sont radicali-

${ }^{28}$ Voir à ce sujet la remarquable synthèse de Christine Leigh Heyrman, Southern Cross. The Beginnings of the Bible Belt, Chapel Hill-Londres, The University of North California Press, 1998, présentée par S. FATH dans «Protestantisme et lien social aux États-Unis», Archives de Sciences Sociales des Religions, 1999, 108 (octobre-décembre), pp. 5-24. Elle montre le basculement d'une culture évangélique "contestataire" et progressiste, au départ (vers 1800) vers une culture conservatrice. Elle attribue largement ce retournement à l'effet de majorité.

${ }^{29}$ Il serait intéressant de tenter un parallèle entre ce "conservatisme" tendanciel de la démocratie locale baptiste et celui de certains cantons suisses où la survalorisation de la démocratie locale peut parfois entretenir, là aussi, un populisme hostile aux évolutions (notamment en terme de droit des femmes).

${ }^{30}$ Lors du grand débat social américain sur l'esclavage dans les années 1840 , la vivacité des discussions fut telle, au sein des dénominations de type « évangélique », qu'elle aboutit à une brutale scission dénominationnelle Nord-Sud chez les méthodistes (1844) aussi bien que chez les baptistes (1845). En revanche, les «Églises-institutions » comme l'Église catholique, l'Église anglicane-épiscopale et l'Église luthérienne ne se sont pas scindées. Dans ces dernières, le débat y fut plus étouffé, plus lent et plus encadré. Relativement vif chez les luthériens, il fut plus discret chez les catholiques : si le pape Grégoire XVI, en 1839, réitéra la condamnation du trafic des esclaves, l'Église catholique n'estimait pas pour autant que l'esclavage soit un péché en soi. Les lettres pastorales des évêques américains, avant la Civil War (1861-1864), sont très discrètes sur le sujet. C'est dans l'Église anglicane-épiscopale que les débats furent les plus timorés avant 1861 . 
sés, que des conflits de valeur ont été médiatisés. Martin Luther-King et nombre de ses proches, mais aussi divers cadres du Ku Klux Klan étaient baptistes, imprégnés de la culture du Bible Belt! Par leur pratique militante, leur structure locale, souple, souvent démocratique, les Églises évangéliques ont réagi avec beaucoup de vivacité aux enjeux du temps, jouant en quelque sorte le rôle de « sismographes du changement social ». 